\title{
Anion influence on the structures of a series of copper(II) metal-organic frameworks
}

Pilar Díaz, Jordi Benet-Buchholz, Ramón Vilar, Andrew J.P. White

\section{Supplementary material}

TGA for metal-organic network $1 \ldots \ldots \ldots \ldots \ldots \ldots \ldots \ldots \ldots \ldots \ldots \ldots . \ldots \ldots$

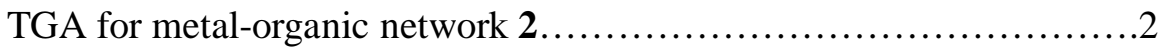

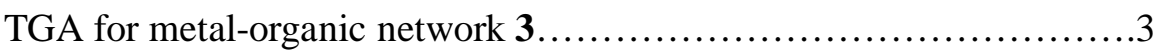

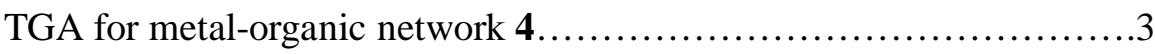

TGA for metal-organic network $5 \ldots \ldots \ldots \ldots \ldots \ldots \ldots \ldots \ldots \ldots \ldots \ldots$

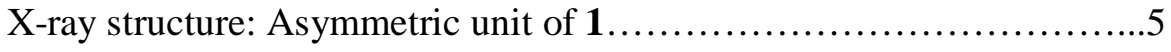

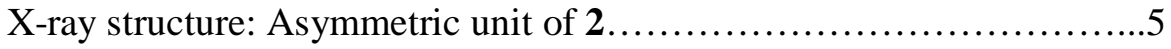

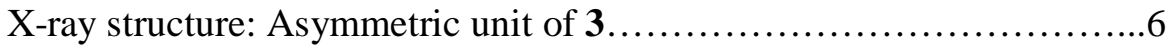

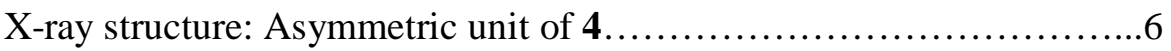

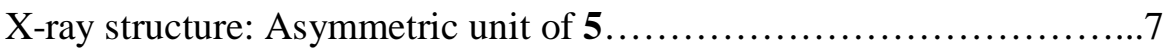

X-ray powder diffraction data of $4 \ldots \ldots \ldots \ldots \ldots \ldots \ldots \ldots \ldots \ldots \ldots \ldots \ldots$ 


\section{Thermo-gravimetric analysis.}

a) TGA for metal-organic network $\mathbf{1}$.

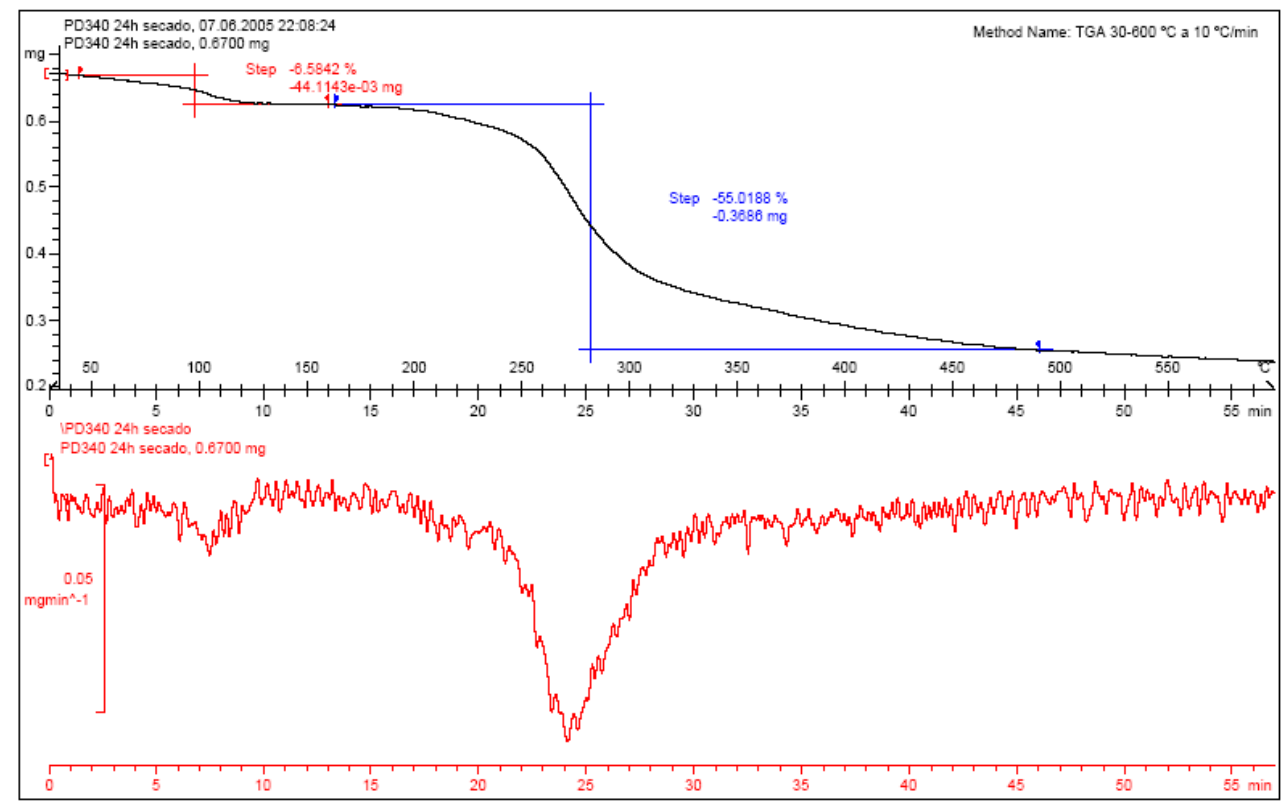

b) TGA for metal-organic network 2 .

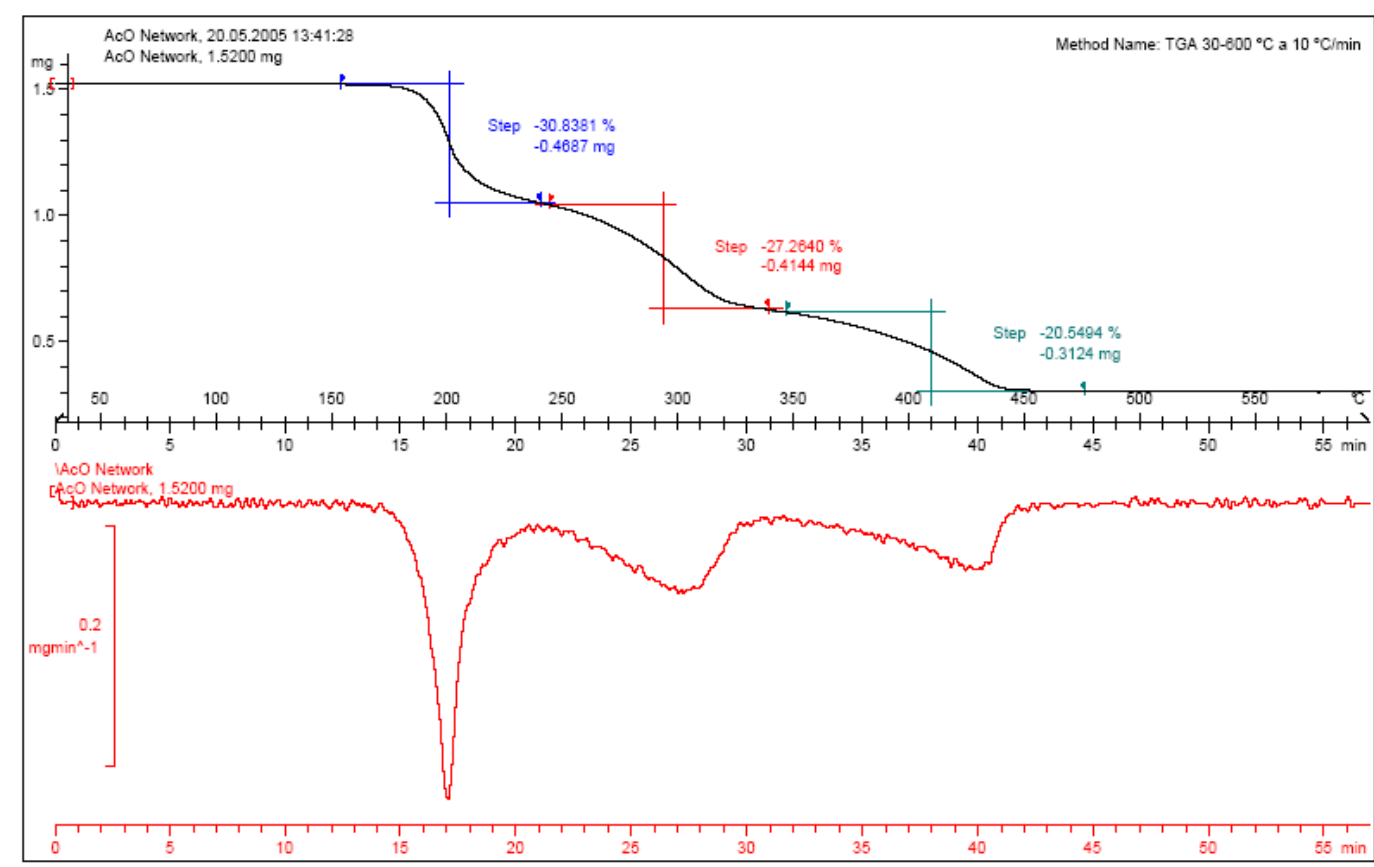


c) TGA for metal-organic network 3 .

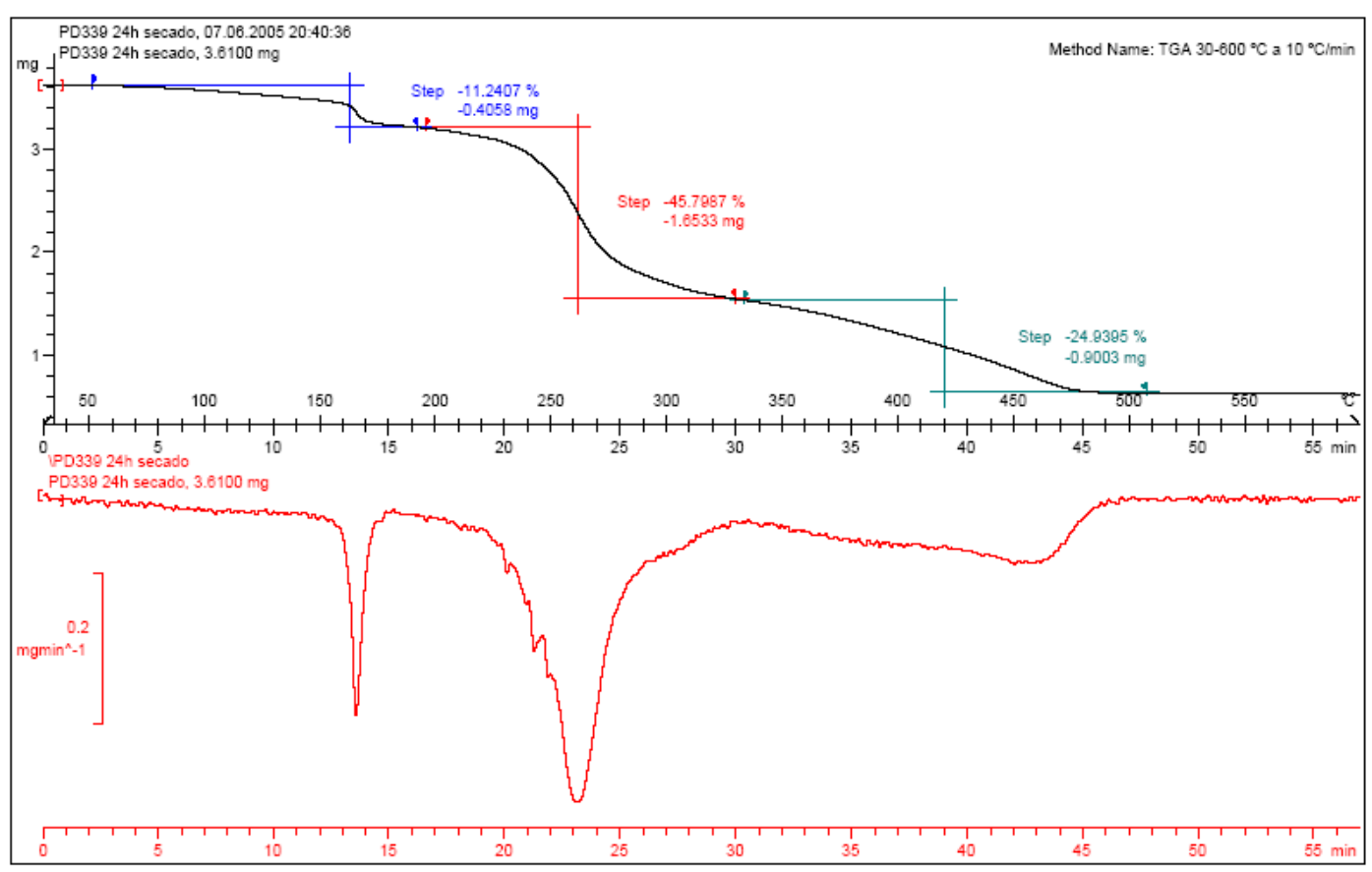

d) TGA for metal-organic network 4 .

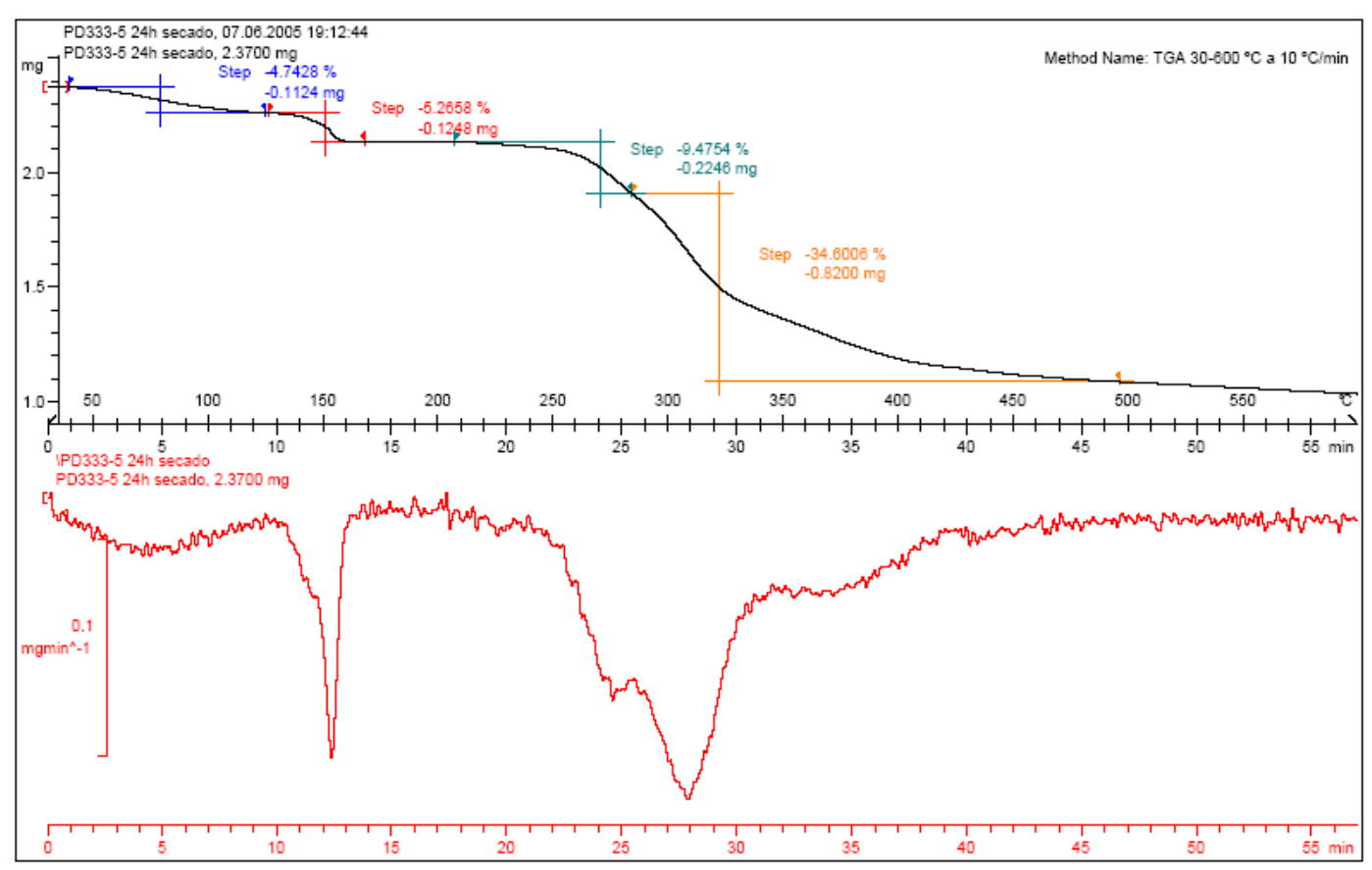


e) TGA for metal-organic network 5 .

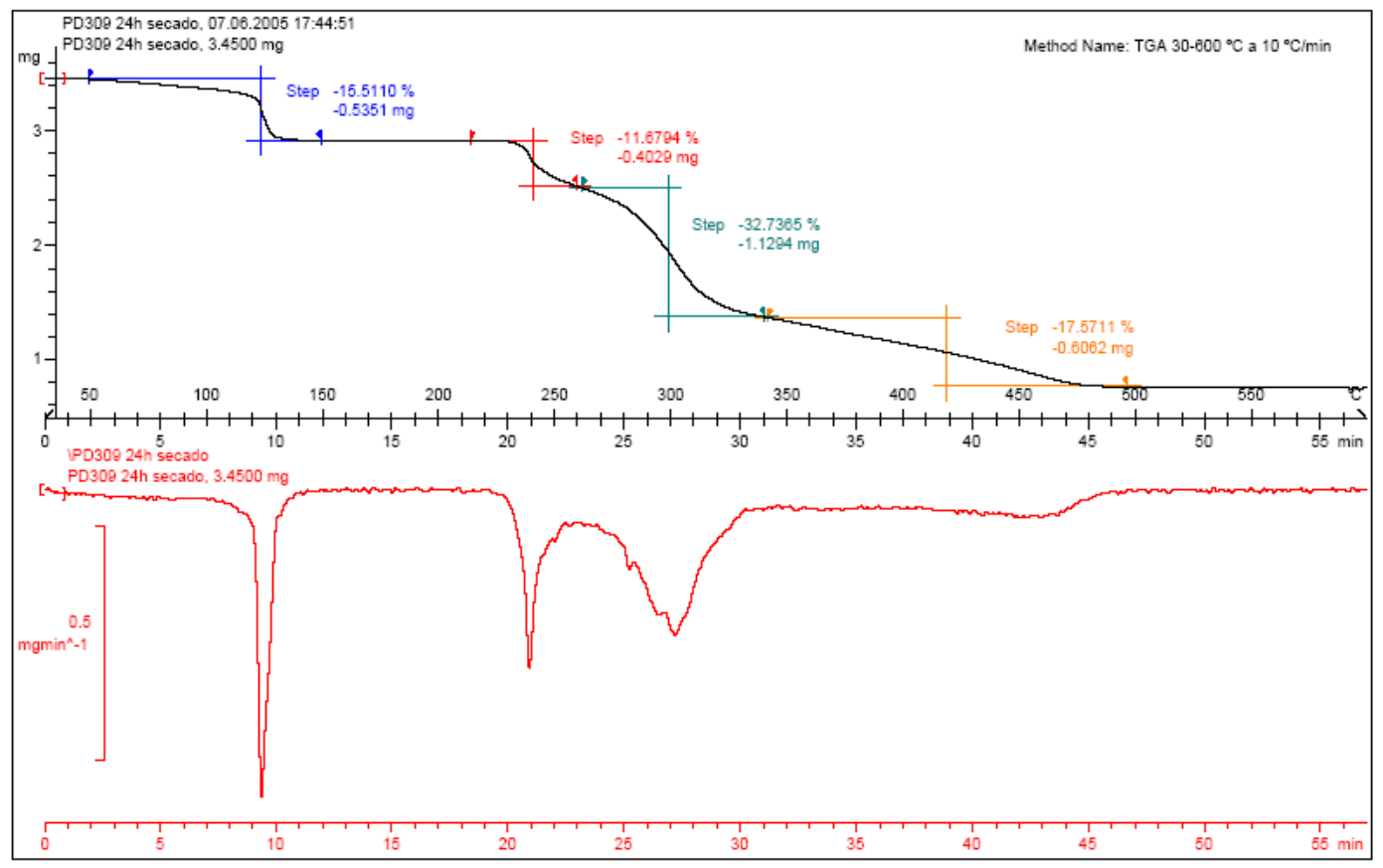




\section{X-ray crystallography}

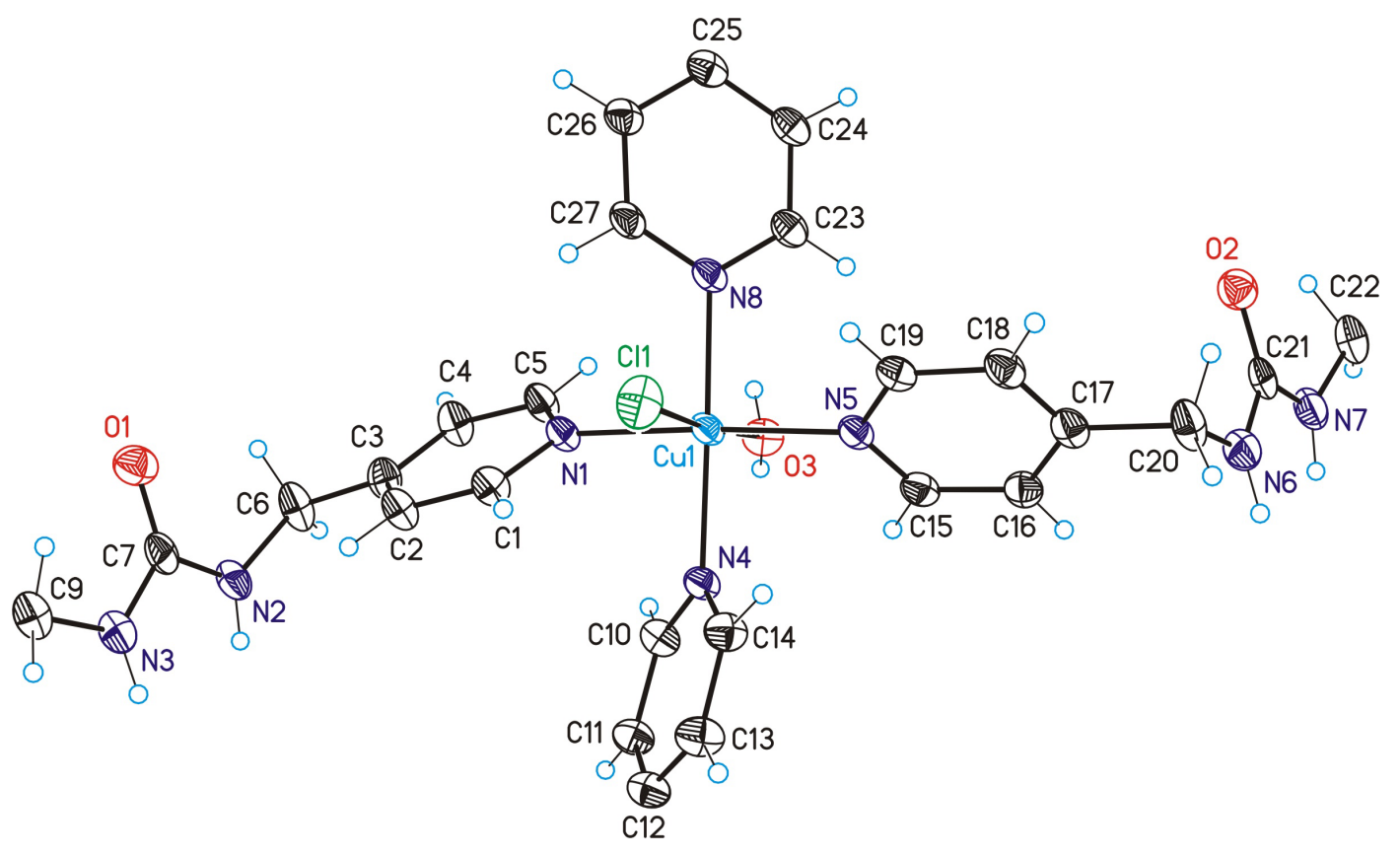

Figure S1 - Asymmetric unit of $\mathbf{1}$

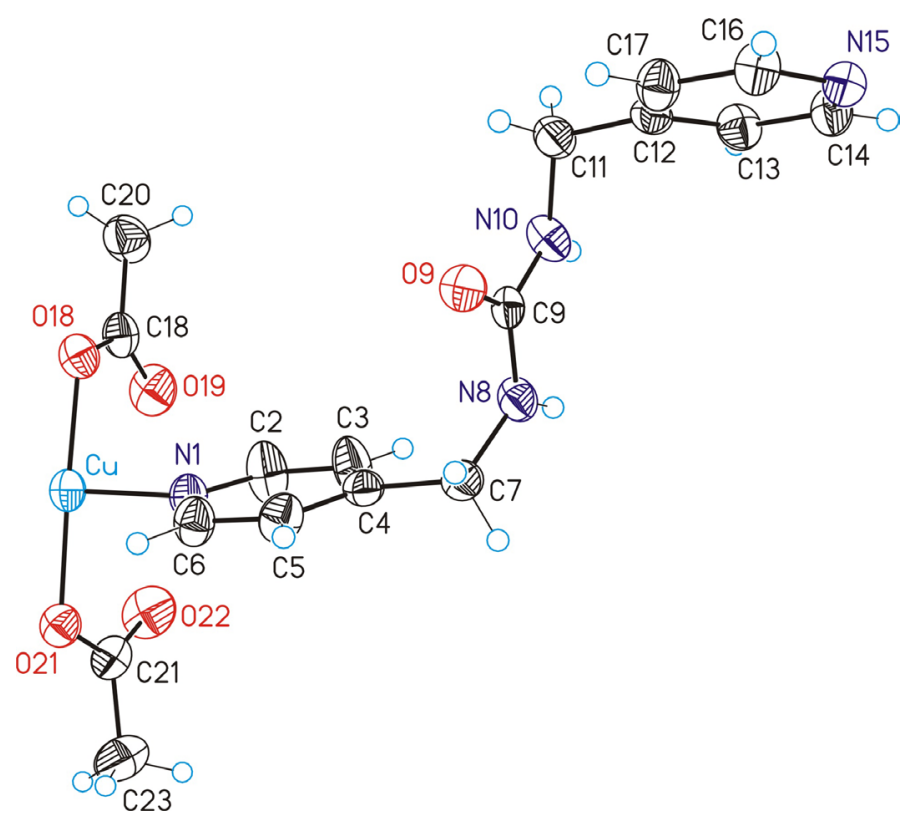

Figure S2 - Asymmetric unit of 2 


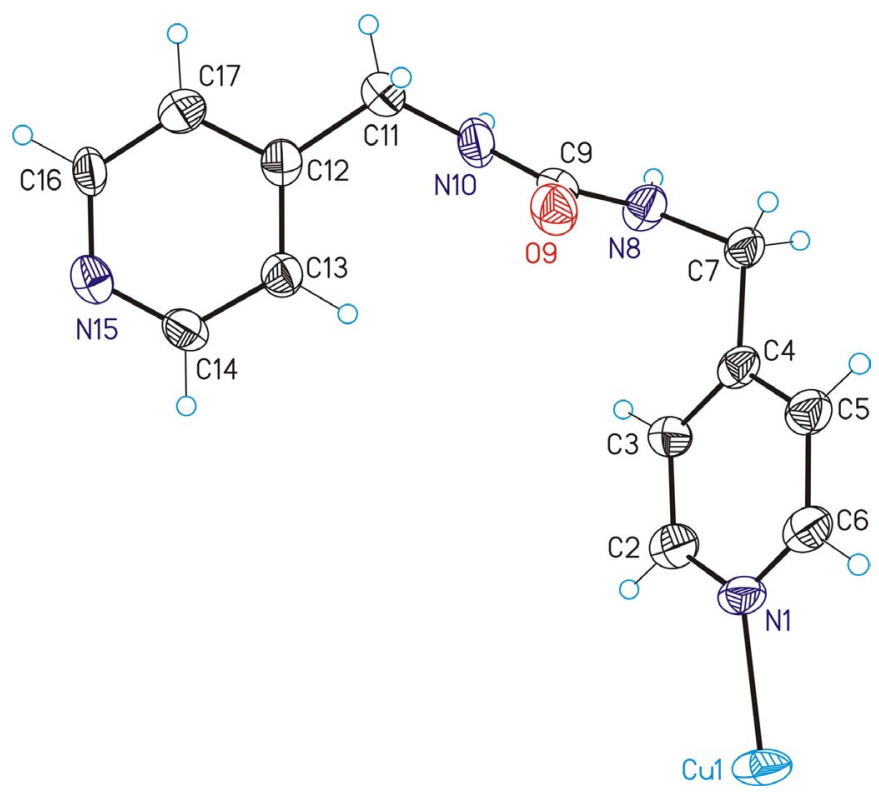

Figure S3 - Smallest fragment of the coordination network 3

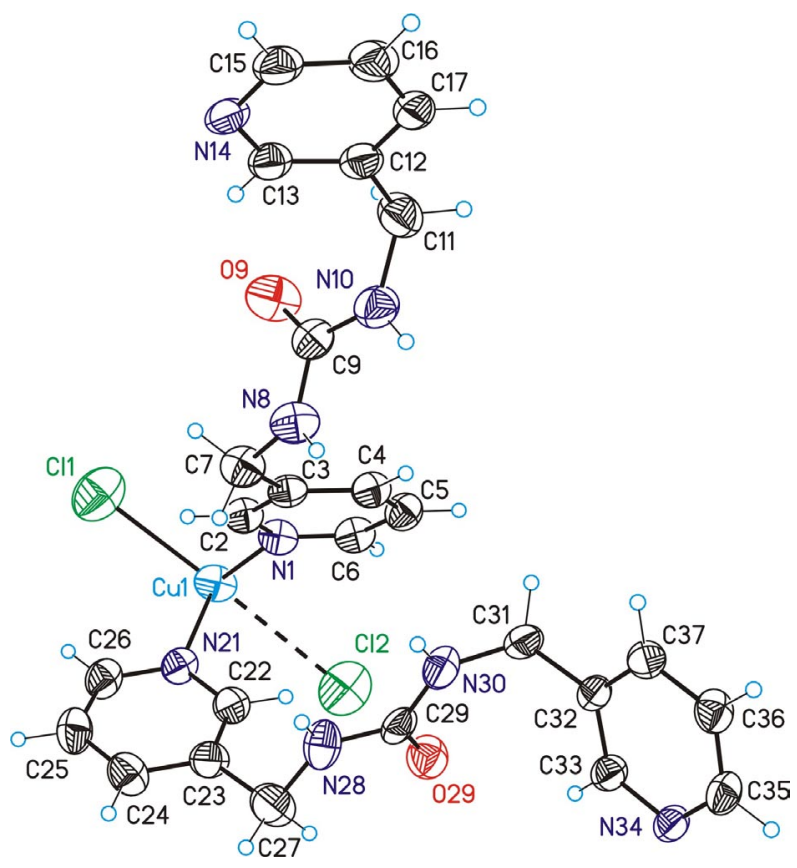

Figure S4 - Asymmetric unit of 4 


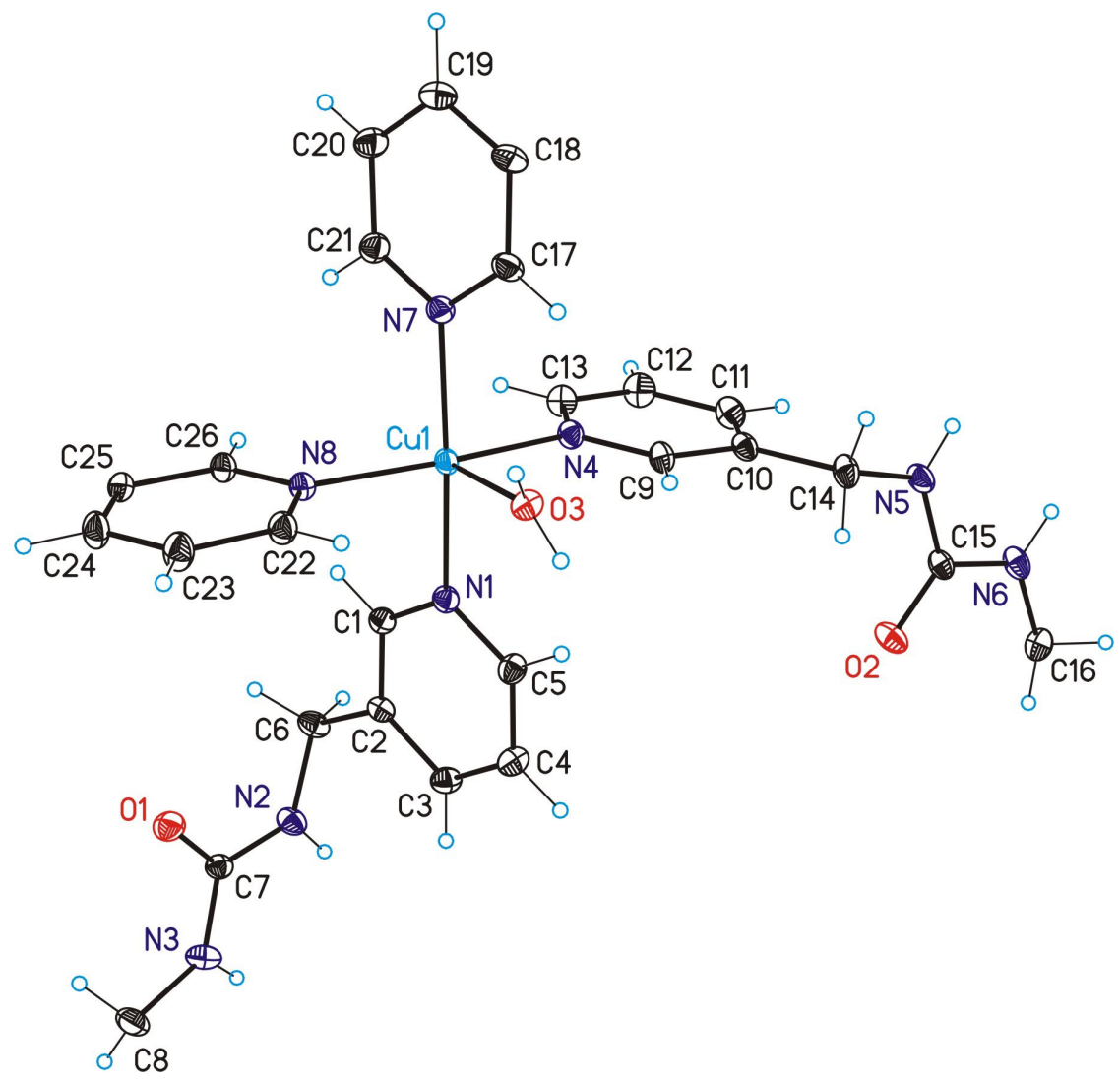

Figure S5 - Asymmetric unit of 5 
Table S1 - Comparison of the diffraction patterns of $\mathbf{4}$ obtained by X-ray powder diffraction (right hand column) and calculated from the single crystal X-ray data (lefthand column).

\begin{tabular}{rrr}
\multicolumn{2}{c}{ Calculated single crystal } & Measured powder diffraction \\
Peak & 2 -theta & 2 -theta \\
1 & 7,22 & 7,11 \\
2 & 8,26 & 8,15 \\
3 & 9,76 & 9,57 \\
4 & 10,16 & 10,09 \\
5 & 11,86 & 11,39 \\
6 & 12,10 & 11,63 \\
7 & 12,58 & 12,48 \\
8 & 13,74 & 14,06 \\
9 & 14,08 & 14,22 \\
10 & 16,16 & 16,08 \\
11 & 16,86 & 16,32 \\
12 & 17,60 & 17,34 \\
13 & 18,20 & 18,11 \\
14 & 18,54 & 18,51 \\
15 & 19,50 & 19,36 \\
16 & 20,38 & 20,13
\end{tabular}

